\title{
CATEGORIZACIÓN DE VARIABLES QUE INCIDEN EN LA CALIDAD DE AGUA, OPERACIÓN Y MANTENIMIENTO DE LOS ACUEDUCTOS RURALES DE LA COMUNIDAD DE EL CALABACITO EN HERRERA, PANAMÁ
}

\author{
Melgar, Yarelis \\ Universidad Tecnológica de Panamá \\ La Chorrera, Panamá \\ yarelis.melgar@utp.ac.pa \\ Deago, Euclides \\ Universidad Tecnológica de Panamá \\ Panamá, Panamá \\ euclides.deago@utp.ac.pa
}

\section{Abstract}

This work made it possible to deepen aspects of water quality, operation and maintenance of four rural aqueducts in El Calabacito, Herrera province. Through the application of surveys, interviews, visits and field inspection, topographic survey and data refinement, it was possible to feed the baseline of this research. The findings show that unmet deficiencies or weaknesses directly or indirectly indicate the quality of water that end users receive. Aspects such as intermittence in the supply, rejection of water disinfection, late payment of fees, little evidence of accounting records, use of aqueduct components, empiricism in decision-making and scarce technical presence and training by the authorities. A categorization of the previously exposed variables is proposed, into internal and external variables according to the type of influence they exert on the systems and based on this information, different alternatives for improvement are proposed for the optimization of the water supply processes of the aqueducts studied.

Keywords: water quality, internal variables, external variables, rural aqueducts, categorization.

\section{Resumen}

Este trabajo permitió profundizar aspectos de calidad de agua, operación y mantenimiento de cuatro acueductos rurales en el Calabacito, provincia de Herrera. Mediante la aplicación 
de encuestas, entrevistas, visitas e inspección de campo, levantamiento topográfico y depuración de los datos, se logró alimentar la línea base de esta investigación. Los hallazgos demuestran que las carencias o debilidades no solventadas indicen de manera directa o indirecta en la calidad de agua que reciben los usuarios finales. Se identificaron aspectos como la intermitencia en el suministro, el rechazo a la desinfección del agua, la morosidad en el pago de tarifas, la poca evidencia de registros contables, sobre uso de los componentes de los acueductos, empirismo en la toma de decisiones y escasa presencia técnica y de capacitación por parte de las autoridades. Se propone una categorización de las variables expuestas anteriormente, en variables internas y externas según el tipo de influencia que ejercen en los sistemas y con base en esta información, se plantean diferentes alternativas de mejora para la optimización de los procesos de suministro de agua de los acueductos estudiados.

Palabras claves: calidad de agua, variables internas, variables externas, acueductos rurales, categorización

\section{INTRODUCCIÓN}

Según cifras del 2019, alrededor de 5 mil acueductos rurales en Panamá son administrados por Juntas Administradoras de Acueductos Rurales (JAAR) [1], quienes bajo la vigilancia del Ministerio de Salud, dotan de agua superficial y subterránea a comunidades periurbanas y comarcales del país [2]. Estos acueductos presentan debilidades mayormente en el diseño, administración, operación y/o mantenimiento de los sistemas, por lo que resulta indispensable diagnosticar y mejorar los procesos de suministro de agua a cargo de las JAAR's, y garantizar un impacto positivo directo a la calidad del agua entregada a las comunidades beneficiadas. No obstante, en la actualidad existe una marcada carencia en la identificación y la amplia discusión del tema. En este trabajo se analizan cuatro acueductos rurales de la comunidad de El Calabacito en la provincia de Herrera. Estos acueductos constan de los siguientes componentes: pozo de extracción, línea de aducción por bombeo, tanque de almacenamiento, red de distribución por gravedad y conexiones domésticas. La calidad del agua de estudio es considerablemente buena; sin embargo, algunos parámetros se encuentran por encima de la norma [3], como es el caso de la conductividad, los sólidos totales disueltos, alcalinidad y coliformes totales [4]. Las principales características de estos sistemas se describen en la tabla 1: 
Tabla 1. Principales características técnicas de los acueductos en estudio [4]

\begin{tabular}{lcccc}
\hline Características Técnicas & La Canoa & Ojo de Agua & El Calabacito & Las Lomas \\
\hline Profundidad de pozo estimada $(\mathrm{m})$ & 37 & 41 & 30 & 19 \\
Caudal de pozo estimado (L/s) & 2,63 & 1,49 & 2,45 & 1,63 \\
Tratamiento de las aguas & No & No & No & No \\
Limpieza al pozo en los últimos 5 años* & No & Sí & No & Sí \\
Capacidad de tanque de almacenamiento (galones) & 5000 & 10000 & 6000 & 5943 \\
Longitud de aducción aproximada (m) & 531 & 520 & 600 & 250 \\
Longitud de conducción aproximada (m) & 489 & 200 & 400 & 300 \\
Material de las tuberías & PVC & PVC & PVC & PVC \\
Diámetro de aducción (pulg) & 2 & 2 & 3 & 2 \\
Diámetro de conducción (pulg) & 2 & 2 & 2 & 2 \\
\hline
\end{tabular}

* A la fecha del estudio

** Línea principal

\section{MÉTODO}

\section{A.Establecimiento de la línea base}

El establecimiento de la línea base para el análisis de los acueductos en estudio se realizó mediante la aplicación de encuestas a miembros de la comunidad, entrevistas a diferentes miembros de la JAAR's, visitas de campo e inspecciones visuales a la infraestructura existente, levantamiento topográfico y depuración de los datos [3]. A través de este último, se identificaron variables relacionadas al diseño, operación y mantenimiento de los sistemas, la administración por parte de las JAAR's y la calidad del agua del área de estudio.

\section{B.Propuesta de categorización}

La propuesta de categorización consistió en la selección y agrupación de variables que, a criterio de los autores, influyen de manera directa o indirecta en la calidad de las aguas del área de estudio. Las variables agrupadas se denominaron: a) Variables internas, las cuales ejercen una influencia directa y b) variables externas, las cuales influyen de forma indirecta en la calidad del agua (tabla 2): 
Tabla 2. Criterios para selección y agrupación de hallazgos en variables internas o externas

\begin{tabular}{lll}
\hline Criterios & Variables internas & Variables externas \\
\hline Definición & $\begin{array}{l}\text { Hallazgos más sobresalientes que influyen } \\
\text { directamente en la calidad del agua del área de } \\
\text { estudio }\end{array}$ & $\begin{array}{l}\text { Hallazgos más sobresalientes que influyen de } \\
\text { forma indirecta en la calidad del agua del área de } \\
\text { estudio }\end{array}$ \\
$\begin{array}{l}\text { Selección y } \\
\text { agrupación }\end{array}$ & $-\quad \begin{array}{l}\text { Ejercen un impacto directo } \\
\text { sistemas }\end{array}$ & $\begin{array}{l}\text { Ejercen un impacto indirecto } \\
\end{array}$ \\
\end{tabular}

\section{RESULTADOS}

Se identificaron aspectos como la intermitencia en el suministro de agua, el rechazo a la desinfección del agua por parte de la comunidad, la morosidad en el pago de tarifas por parte de los usuarios, la poca evidencia de registros contables por parte de la administración, sobre uso de las instalaciones y componentes de los acueductos, empirismo en la toma de decisiones; inclusive, la escasa presencia técnica y de capacitación por parte de las autoridades. La tabla 3 muestra la propuesta de categorización.

Tabla 3. Propuesta de categorización de hallazgos

\begin{tabular}{|c|c|c|c|}
\hline No. & Categorías & Variables internas & Variables externas \\
\hline 1. & $\begin{array}{l}\text { Diseño y } \\
\text { mantenimiento }\end{array}$ & $\begin{array}{l}\text { - Escaso monitoreo y conocimiento } \\
\text { acerca del funcionamiento hidráulico } \\
\text { de los sistemas } \\
\text { - Sobreuso de los componentes de los } \\
\text { sistemas por encima de la vida útil }\end{array}$ & $\begin{array}{l}\text { Poco tecnicismo en la toma de decisiones } \\
\text { sobre el funcionamiento y operación de los } \\
\text { sistemas } \\
\text { - Conocimiento empírico por encima del } \\
\text { conocimiento técnico } \\
\text { Escasa presencia y capacitación por parte } \\
\text { de autoridades competentes }\end{array}$ \\
\hline 2. & $\begin{array}{l}\text { Operación de los } \\
\text { sistemas }\end{array}$ & $\begin{array}{l}\text { - Intermitencia en el suministro de agua } \\
\text { Decisión de no desinfección del agua } \\
\text { por parte de los miembros de la } \\
\text { comunidad }\end{array}$ & \\
\hline 3. & $\begin{array}{l}\text { Administración } \\
\text { JAAR's }\end{array}$ & & $\begin{array}{l}\text { - } \quad \text { Morosidad en el pago de tarifas } \\
\text { - } \quad \text { directiva de las JAAR's } \\
\text { - } 2 / 4 \text { JAAR's cuentan con personería jurídica }\end{array}$ \\
\hline
\end{tabular}

Para solventar estos hallazgos se recomienda implementar las siguientes acciones de mejora:

- Realizar un estudio profundo para conocer el estado de los componentes del sistema y reemplazar los que hayan sobrepasado su vida útil.

- Aumentar la presencia de autoridades locales, MINSA y organismos internacionales en jornadas de capacitación sobre manejo de sistemas de acueductos rurales. Se 
debe reforzar el conocimiento empírico con la toma de decisiones con carácter técnico por parte de las JAAR's.

- Se recomienda que el suministro de agua sea continuo para evitar la contaminación de las redes de distribución [5].

- El MINSA debe ejercer rol de capacitador hacia la comunidad, impartiendo docencia acerca de enfermedades de transmisión hídrica y la importancia de desinfectar el agua como tratamiento.

- Se recomienda la implementación de acuerdos de pago para reducir la morosidad por parte de los usuarios de los sistemas.

- Se recomienda llevar registro financiero de las compras, pagos por servicios de reparación, cobro de tarifas, listados de morosos y otros.

- Se recomienda que todas las JAAR's cuenten debidamente con personería jurídica, ya que esta condición legal les permite ejecutar acciones como cortes de servicios a usuarios morosos, entre otros beneficios como fondos de financiamiento nacional e internacional para la mejora de sus sistemas.

\section{CONCLUSIONES}

La propuesta de categorización permite seleccionar aspectos de diseño, operación, mantenimiento y administración de los acueductos del caso de estudio conforme al impacto que, a criterio de los autores, estos aspectos suponen en la calidad del agua. Los resultados demuestran que el diseño y mantenimiento de los componentes representa un factor predominante, ya que pueden influir tanto directa como indirectamente. Por su parte, las decisiones referentes a la operación de los sistemas por parte de las JAAR's logran influir directamente en la calidad del agua; mientras que las actividades propias de la administración financiera de las JAAR's, influyen indirectamente.

\section{Referencias}

[1] G. Cárdenas Castillero, "El agua del otro Panamá," La Prensa, Panamá, República de Panamá, p. 1, Sep. 06, 2019.

[2] M. Diaz, "Las Juntas Administradoras de Acueductos Rurales -JAAR-, aportando al Derecho Humano al Agua en PANAMÁ," Panamá, 2014. [Online]. Available: http://alianzaporelagua.org/documentos/ Gestion_Comunitaria_Agua/Panama.pdf.

[3] Ministerio de Comercio e Industrias, "Resolución No. 35 de 6 de mayo de 2019," Panamá, República de Panamá, 2019. doi: 10.1016/j.ophtha.2009.04.026.

[4] Y. Melgar and A. Vergara, "Diagnóstico y mejoras al sistema de acueducto rural de las comunidades de Ojo de Agua, Las Lomas, La Canoa y El Calabacito," Universidad Tecnológica de Panamá, 2020.

[5] C. I. Gonzalez, J. Erickson, K. A. Chavarría, K. L. Nelson, and A. Goodridge, "Household stored water quality in an intermittent water supply network in Panama," J. Water, Sanit. Hyg. Dev., pp. 1-11, 2020, doi: 10.2166/washdev.2020.156. 


\section{Autorización y Licencia CC}

Los autores autorizan a APANAC XVIII a publicar el artículo en las actas de la conferencia en Acceso

Abierto (Open Access) en diversos formatos digitales (PDF, HTML, EPUB) e integrarlos en diversas plataformas online como repositorios y bases de datos bajo la licencia CC:

Attribution-NonCommercial-ShareAlike 4.0 International (CC BY-NC-SA 4.0) https://creativecommons. org/licenses/by-nc-sa/4.0/.

Ni APANAC XVIII ni los editores son responsables ni del contenido ni de las implicaciones de lo expresado en el artículo. 